\title{
Energy selective neutron radiography in material research
}

\author{
Lidija Josic • A. Steuwer $\cdot$ E. Lehmann
}

Received: 24 March 2009 / Accepted: 24 February 2010 / Published online: 20 March 2010

(c) Springer-Verlag 2010

\begin{abstract}
Energy selective neutron radiography was performed to describe a complex structure in polycrystalline materials. Experiments were performed with currently the highest energy and spatial resolutions achieved simultaneously, by employing a double crystal monochromator for selecting narrow energy bands from the initially polychromatic neutron beam and the neutron absorbing scintillator screen coupled with the cooled CCD camera as a detection system. It was shown that the detailed structure of the welded steel sample can be visualized and quantified by performing energy selective neutron imaging in the cold energy range, where elastic coherent scattering dominates the total cross section of the sample, showing characteristic Bragg edges. With the maps of crystallographic orientations over the sample area of $\sim 2 \times 2 \mathrm{~cm}^{2}$ and thickness $\sim 11.2 \mathrm{~mm}$, obtained directly from radiographs, the complex structure was energy resolved with a spatial resolution of $\sim 50 \mu \mathrm{m}$.
\end{abstract}

\section{Introduction}

In conventional neutron radiography and tomography, mostly polychromatic thermal and cold neutron beams are used [1-4]. Although having the advantage of high neutron

L. Josic $(\bowtie) \cdot$ E. Lehmann

Spallation Neutron Division, Paul Scherrer Institut (PSI),

Villigen, Switzerland

e-mail: lidija.josic@psi.ch

Fax: +41-563103131

A. Steuwer

ESS Scandinavia, Lund, Sweden fluxes and thus low exposure times, provided image contrast is an average over a broad energy range. As a consequence the energy depended characteristics of the sample are not visible. In the cold range, the image contrast is more pronounced because of the higher energy averaged value of the neutron total cross sections that attenuates the initial beam more effectively in comparison to the thermal range. Narrowing the energy band of the incident beam further increases the image contrast significantly, especially near the Bragg edges (Fig. 1).

Energy selective neutron imaging of crystalline materials in the cold energy range has firstly been performed by applying a velocity selector device in both radiography and tomography modes [5-7]. Although the energy resolution was relatively poor $(\Delta \lambda / \lambda \sim 30 \%)$, it was demonstrated that by choosing specific neutron energies the image contrast between different materials within a sample can be increased. Material with the same attenuation coefficient at two different energies, for example, can be made transparent on the image obtained by division of transmission images taken at those two energies. In general, choosing a single energy where the products of the attenuation coefficient and thickness for different materials differ, increases the image contrast. Further improvements in the energy resolution have been achieved at the CONRAD cold neutron beam line by an installation of a double monocrystal monochromator device [8] and at ICON cold neutron beam line by using a velocity selector device of a higher performance and a transmission based crystal monochromator currently under development. The first experiments that managed to image the crystalline structure have been performed at ICON and were later confirmed in experiments at ISIS, UK pulsed source [9]. The temporal/spatial resolution was high enough to detect the presence of textures in the welded steel sample. It has been demonstrated that a neutron imaging technique has the best 
Fig. 1 Total neutron cross sections $(\sigma)$ for seven different polycrystalline materials derived from the CRIPO code [29]. The dominant elastic coherent scattering with characteristic Bragg edges enables energy selective neutron imaging with the highest possible contrasts near Bragg edges

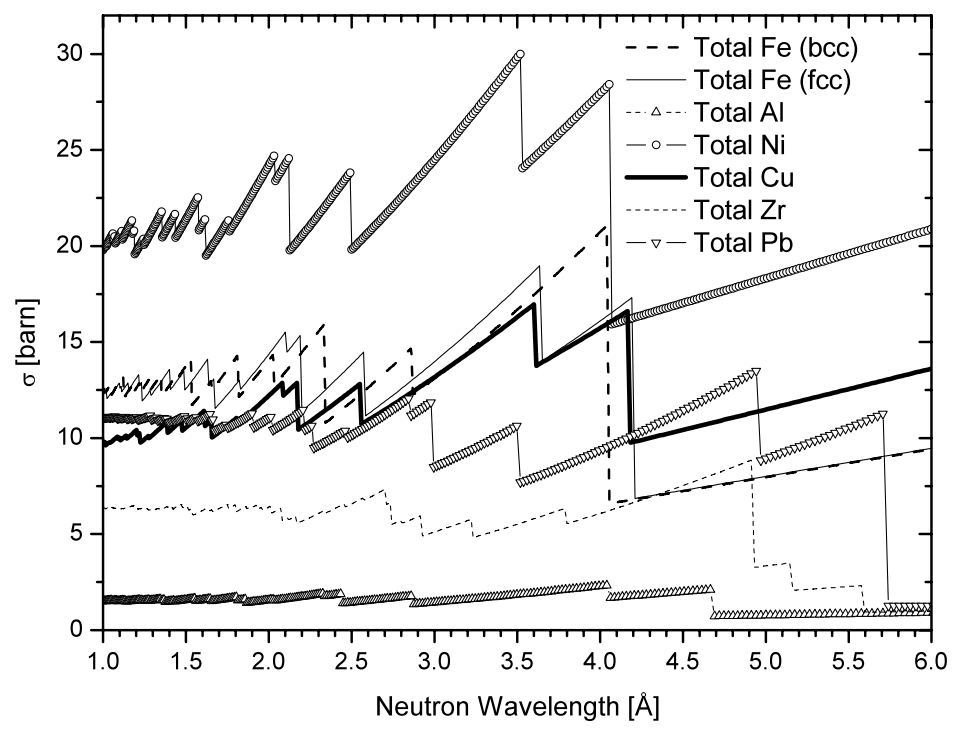

possible performance to map the crystalline structure over the whole sample area and thickness with the highest possible spatial resolution. In addition, a digital detection system generally enables high performance data quantification. The Bragg transmission method employed at ISIS in the standard set up is, on the other hand, capable to measure crystalline structure parameters such as the crystal lattice spacing, textures, lattice constants, residual strains and phase selective contrast [10-14] with an uncertainty of $0.0001 \AA$ [10] but does not provide a high spatial resolution over the sample area. Such a high precision is influenced only by the temporal resolution of the counting detector because the Bragg edge positions are formed with the backscattered neutrons when Bragg angles reach $90^{\circ}$ values for specific crystallographic planes of the sample. The best reported spatial resolution of $\sim 15 \mu \mathrm{m}$ was achieved with neutron sensitive microchannel plates (MCP) where the neutron capture process is preserved within one or two channels of the typical diameter of $6-10 \mu \mathrm{m}$ and the wall thickness of 2-3 $\mu \mathrm{m}$ [15]. Nevertheless, an MPC device is not currently available for the standard user program at neutron imaging beam lines. In this work, results of the latest simultaneous improvement in energy and spatial resolutions applied to the analysis of the complex structure in the welded steel sample are presented. In Sect. 2, an introduction to the physics of neutron scattering and formation of transmission signals for polycrystalline materials is given. Section 3 describes the neutron radiography experiment followed by the summary of experimental methods for narrowing the energy bands in Sect. 4. Section 5 summarizes experimental results together with the data interpretation and discussion, and Sect. 6 concludes.

\section{Total scattering cross section and transmission signal in neutron imaging}

\subsection{Total scattering cross section of neutrons}

When a neutron interacts with an atom, it can be either absorbed or scattered dominantly by the atomic nucleus. The scattering process is determined by the neutron scattering length $b$, which is an unique quantity of the atom. If neutrons interact with materials, the total cross section $\sigma(\lambda)$ consists of coherent and incoherent, elastic and inelastic scattering contributions and absorption [16]:

$$
\begin{aligned}
\sigma(\lambda)= & \bar{\sigma}_{\mathrm{coh}}\left(S_{\mathrm{coh}}^{\mathrm{el}}(\lambda)+S_{\mathrm{coh}}^{\mathrm{inel}}(\lambda)\right) \\
& +\bar{\sigma}_{\mathrm{inc}}\left(S_{\mathrm{inc}}^{\mathrm{el}}(\lambda)+S_{\text {inc }}^{\text {inel }}(\lambda)\right)+\sigma_{\mathrm{abs}}(\lambda)
\end{aligned}
$$

where $\bar{\sigma}_{\text {coh }}$ and $\bar{\sigma}_{\text {inc }}$ are the averaged coherent and incoherent scattering cross sections per nucleus in the high energy limit and $S(\lambda)$ functions that contain the structure and dynamics of the material. In particular, only $S_{\mathrm{coh}}^{\mathrm{el}}(\lambda)$ includes structural parameters while other functions continuously increase or decrease with $\lambda$. It can be seen, for example, in the resolved total cross section of $\mathrm{Pb}$ [17] or $\alpha$-Fe [16].

For any type of the perfect structure, the elastic coherent scattering cross section is given by $[18,19]$

$\sigma_{\mathrm{coh}}^{\mathrm{el}}(\lambda)=\frac{\lambda^{2}}{2 V_{0}} \sum_{d_{\vec{h}}=0}^{2 d_{\vec{h}}<\lambda}\left|F_{\vec{h}}\right|^{2} d_{\vec{h}}$

with the structure factor [16]: 


$$
\begin{aligned}
F_{\vec{h}}= & \varpi(\vec{h}) \sum_{i} o_{i} b_{i} \\
& \times \exp \left(2 \pi i \vec{h} \cdot \vec{x}_{i}\right) \exp \left(-\frac{3 h^{2} \varphi_{1}\left(\Theta_{i}\right)}{2 M_{i} k_{\mathrm{B}} \Theta_{D, i} d_{\vec{h}}^{2}}\right)
\end{aligned}
$$

where $V_{0}$ is the volume of the unit cell, $h$ and $k_{\mathrm{B}}$ Planck's and Boltzmann's constants, $M_{i}$ and $\Theta_{\mathrm{D}, i}$ the mass and Debye temperature of the $i$ th nucleus, $\Theta_{i}=T_{i} / \Theta_{\mathrm{D}, i}$, and $o_{i}, b_{i}$ and $\vec{x}_{i}$ the occupation factor, scattering length and position of the $i$ th nucleus. The function $\varphi_{1}\left(\Theta_{i}\right)$ is described in detail in the reference [19]. $\vec{h}=\vec{\tau} / 2 \pi, \varpi(\vec{h})$ and $d_{\vec{h}}=1 /|\vec{h}|$ are the reciprocal vector, its multiplicity and spacing of the particular set of crystallographic planes $(h, k, l)$ conventionally used in crystallography $\left(\vec{h}=h \vec{a}^{*}+k \vec{b}^{*}+l \vec{c}^{*}\right.$ and $a, b$ and $c$ are the lattice constants). It can be shown that a significant scattering intensity is obtained only if the neutrons are scattered coherently. This is fulfilled if the intensities of neutron momentum transfer and reciprocal vectors are equal:

$\left|\vec{k}^{\prime}-\vec{k}\right|=2|\vec{k}| \sin \theta=|\vec{\tau}|=2 \pi|\vec{h}|=2 \pi / d_{\vec{h}}$

where $\vec{k}$ and $\vec{k}^{\prime}$ are the incident and scattered neutron wave vectors and $\theta$ the scattering angle. Because of the relation $|\vec{k}|=2 \pi / \lambda$ between the neutron wave vector and wavelength $(\lambda)$, the former equation transforms to Bragg law:

$2 d_{h k l} \sin \theta=\lambda$.

In our image interpretation, we can directly make use of this Bragg law relation in both qualitative and quantitative manners for the first time.

\subsection{Formation of the transmission signal in neutron imaging}

In neutron transmission experiments, crystallographic parameters are obtained by an analysis of the Bragg transmission patterns. Those patterns show a set of peaks superimposed to the continual background. According to the Bragg law (5), different sets of crystallographic planes contribute to the transmission pattern and each single $(h, k, l)$ set only in the wavelength range from $\lambda=0$ to $\lambda=2 d_{h k l}=\lambda^{\max }$. $\lambda^{\max }$ is named the Bragg edge or Bragg cut off because above that value reflections from a corresponding $(h, k, l)$ set are not anymore possible. As a consequence, a sharp increase in the transmission appears at each $\lambda^{\max }$ value. In the monocrystal transmission pattern, Bragg peaks are sharp dips that can be used for the determination of the crystal orientation. If the sample is powder or polycrystal with randomly oriented crystallites, the transmission between the Bragg edges is continuous. In the quantification sense, the height of the $(h, k, l)$ Bragg edge provides the number of crystallites with the $(h, k, l)$ plane perpendicular to the incident neutron beam direction and the transmission left from the Bragg edge in the wavelength scale, the number of crystallites with possible $(h, k, l)$ reflections under the angle:

$\alpha_{h k l}=\frac{\pi}{2}-\arcsin \left(\frac{\lambda}{2 d_{h k l}}\right)$.

For the textured polycrystal those numbers of crystallites change, and the elastic coherent scattering cross section (2) changes to:

$\sigma_{\mathrm{coh}}^{\mathrm{el}}(\lambda)=\frac{\lambda^{2}}{2 V_{0}} \sum_{d_{\vec{h}}=0}^{2 d_{\vec{h}}<\lambda}\left|F_{\vec{h}}\right|^{2} d_{\vec{h}} R\left(\alpha_{\vec{h}}\right)$

which further can provide the ratio $R\left(\alpha_{\vec{h}}\right)$ of crystallites numbers in textured and non-textured sample in the cone of opening $2 \alpha_{\vec{h}}$ around the incident beam direction.

Finally, the Bragg edge position on the wavelength scale determines the lattice spacing $d_{h k l}=\lambda^{\max } / 2$ and thus the lattice constants $a, b$ and $c$.

\section{Neutron radiography experiment}

In neutron imaging the standard experimental set up consists of the neutron source, sample and neutron detector aligned along the same straightforward direction with the 2D detector positioned perpendicularly. Neutrons are produced either by fission processes in the nuclear reactors or by spallation processes in the novel neutron sources. Both types of sources are capable of producing either continuous neutron beams or neutron pulses with the time structure included for the imaging beam lines. Further, neutrons with parallel velocity vectors directed to the sample and detector are extracted by curved neutron guides (to lower the background from $\gamma$ rays emitted by the source), flight tubes, or in a combination, which performs collimation, e.g. the absorption of non-parallel directed neutrons. Only neutrons that were transmitted through the sample reach the detector in ideal experimental conditions, while neutrons absorbed or scattered by the sample are removed from the incident neutron beam or pulse. From the detector side several solutions are in the standard use [20, 21]. In order to be detected, neutrons are firstly absorbed by the most common isotopes, B-10, Gd or Li-6, and the secondary ionizing radiation produced is effectively detected. Described set ups are currently adopted on the available imaging beam lines (NEUTRA, ICON, CONRAD, ANTARES and other).

The measurable quantity in the neutron radiography experiment is the neutron beam intensity behind the sample $I(x, y)$ for each detector pixel $(x, y)$, weighted by the energy depended detector efficiency $\varepsilon(E)$, and averaged over the neutron energy spectrum at the detector and sample thickness in the beam direction $(z)$. According to the exponential 
attenuation law, $I(x, y)$ can be evaluated as:

$$
\begin{aligned}
I(x, y)= & \int I_{0}(x, y, E) \varepsilon(E) \\
& \times \exp \left(-\int \Sigma(x, y, z, E) d z\right) d E
\end{aligned}
$$

where

$$
\Sigma(x, y, z, E)=n(x, y, z) \sigma(E)
$$

is the attenuation coefficient, $n(x, y, z)$ the number of sample atoms per unit volume and $\sigma(E)$ the total neutron cross section.

Measuring the beam intensity $I_{0}(x, y)$ without the sample in the beam:

$I_{0}(x, y)=\int I_{0}(x, y, E) \varepsilon(E) d E$,

transmission is readily obtained as a ratio:

$T(x, y)=\frac{I(x, y)}{I_{0}(x, y)}$.

This further enables an evaluation of the attenuation coefficient $\Sigma(x, y)$ averaged over the energy spectrum and sample thickness $z$ according to the exponential attenuation law:

$\Sigma(x, y)=-\frac{\ln (T(x, y))}{z}$.

Several effects may disturb the pure transmission signal. The dominant one is a detection of not only transmitted neutrons but also neutrons scattered from the sample at small sample detector distances [22, 23]. This means that the total cross section $\sigma(E)$ needs to be well determined in order to subtract the scattering component from the radiograph. The effect can be especially significant for the monochromatic neutron beams in the cold energy range where the elastic coherent scattering dominates the total cross section. Numerical procedures based on Monte Carlo simulations of the neutron radiography experiment are usually used for an estimate of the scattering component by using analytically evaluated cross sections as an input [24, 25]. After the correction, the pure transmission image is obtained which enables the full image contrast on the broad gray level scale and the quantification.

\section{Experimental methods for energy selective imaging}

\subsection{Velocity selector device}

Velocity selector is a time of flight device that transmits neutrons of wavelengths in narrowed bands from the initial polychromatic neutron beam (Fig. 2). It is made of absorbing helical blades inserted in the rotor with the changeable rotation frequency and acts as a moving collimator in the wavelength space. Only neutrons that manage to pass between the blades, in the time interval determined by the rotation frequency, are transmitted and contribute to the narrowed wavelength distribution. Those transmission distributions can be approximated by Gaussian functions in a neutron velocity $(v)$ space with a width $\Delta v$ [26]

$\Delta v=\frac{v_{0} \sqrt{\alpha^{2}+\beta^{2}}}{\operatorname{tg} \gamma}$

and a maximal transmission value $T_{m}$

$T_{m}=\frac{T_{m}^{0} \beta}{\sqrt{\alpha^{2}+\beta^{2}}}$

where $T_{m}^{0}$ is the maximal transmission for the neutrons with their velocity vectors parallel to the rotor axis, $\alpha$ the divergence of the incident neutron beam, $\beta$ the divergence determined by two adjacent blades and $\gamma$ the tilted angle between blades and the incident beam direction. Most of the velocity selectors provide the wavelength bands of the width $\Delta \lambda / \lambda \geq 10 \%$ where $\lambda$ corresponds to the $T_{m}$ value. On the neutron imaging beam lines, a velocity selector has been established as a satisfactory good option for a variety of investigations [2, 5-7] because of its relatively high-transmitted neutron flux, a reasonable high energy resolution and a high available spatial resolution of $50 \mu \mathrm{m}$ determined by a good beam collimation and advanced imaging detectors. Because of the possibility for the installation of additional devices for increasing the wavelength resolution (e.g. monocrystal monochromators) this method can be improved to high performance in the energy/spatial resolution.

\subsection{Pulsed neutron sources}

Pulsed sources generate neutron pulses of tens of microseconds with the highest available neutron fluxes in the order of $10^{16} \mathrm{n} / \mathrm{s} \cdot \mathrm{cm}^{2}$ (LANSCE, USA; ISIS, UK) and $10^{17}$ $\mathrm{n} / \mathrm{s} \cdot \mathrm{cm}^{2}$ (SNS, USA; J-PARC, Japan), which are detected in the counting mode with the high temporal resolution. Detecting arrival time spectra of pulses from the source to the detector, neutron wavelengths are obtained from the de Broglie relation:

$\lambda=\frac{h}{m v}=\frac{h t}{m L}$

where $h$ is Planck's constant, $m$ the neutron mass and $t$ its time of flight over the source-detector distance $L$. The wavelength resolution at pulsed sources is determined by the uncertainty in time and position at which the neutron starts its flight from the source moderator to the detector and the 
Fig. 2 Velocity selector device: perspective view (left) and top view (right). Narrowed energy band is formed from the polychromatic beam by transmission of neutrons that manage to pass between the rotating blades
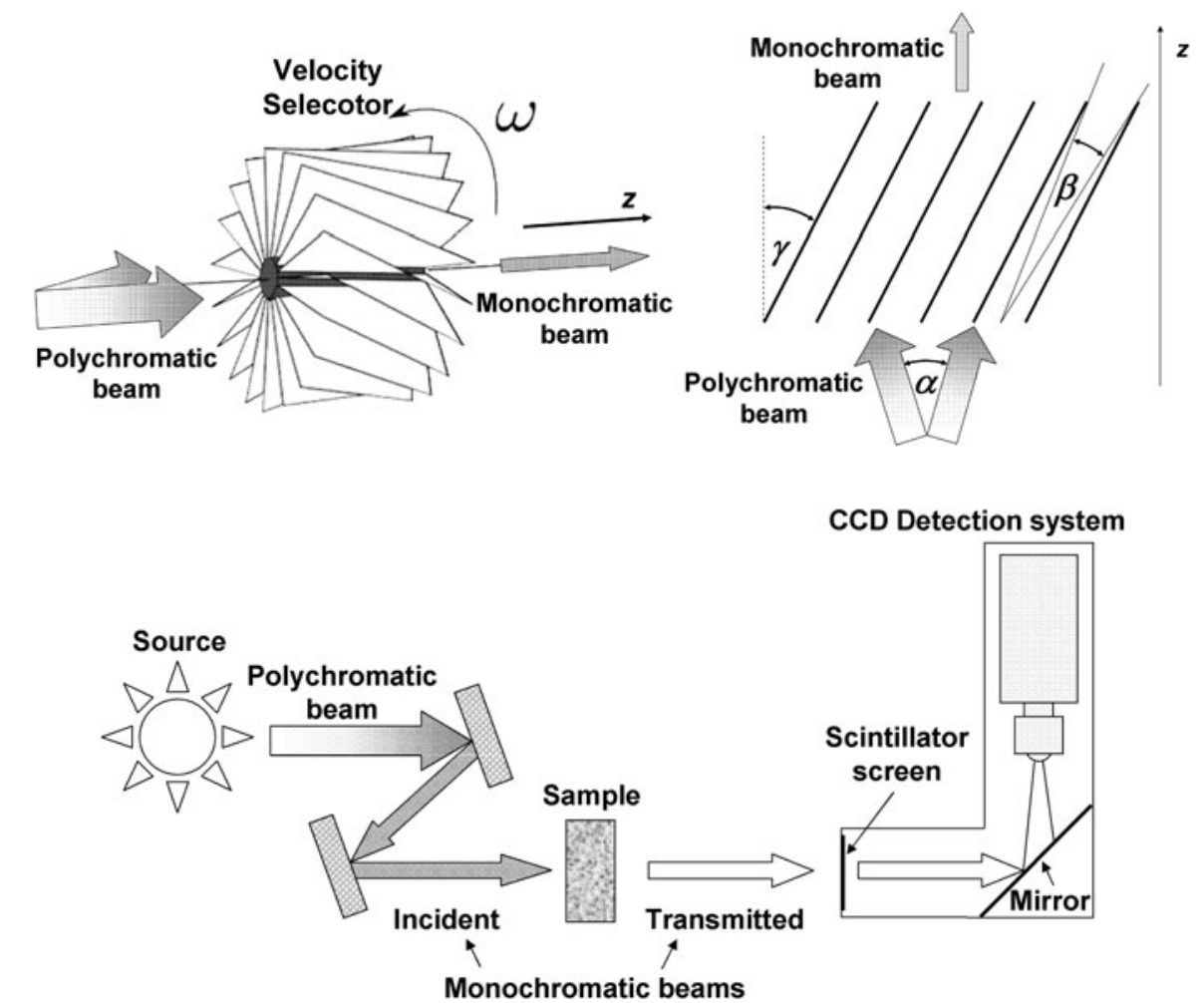

CCD Detection system
Fig. 3 Radiography set up with a double monocrystal monochromator device. Narrowed energy band in the incident beam direction is formed from the polychromatic beam by reflections of the wanted wavelength from two monocrystals according to the Bragg law. Transmitted beam after the sample is absorbed by the scintillator and produced light is recorded by the CCD camera via reflecting mirror and camera optics width of the detector time channel [10]. As a sub method for selecting narrow neutron energy bands, a chopped continuous neutron current can be applied using the same principle. An installation of neutron imaging beam lines at pulsed neutron sources would be an advanced improvement because of a merge of the highest available neutron flux and high temporal resolution with a high spatial resolution of the imaging detector systems [27]. The current low spatial resolution of 1 to $2 \mathrm{~mm}$ at pulsed sources is restricted by the used detector systems.

\subsection{Reflection (diffraction) based monocrystal monochromator device}

Monocrystal monochromators select the wanted wavelength bands from the initial polychromatic neutron beam by rotating a monocrystal according to the Bragg law. The wavelength resolution in such devices is determined by the mosaicity of the crystal and divergence of the initial polychromatic beam. In a double monocrystal monochromator device (Fig. 3) installed at CONRAD, HMI [8], a second monocrystal is implemented in order to reflect the selected monochromatic beam into the initial beam direction from the source. This device provides wavelength bands of relatively low widths $(10 \%>\Delta \lambda / \lambda>1 \%)$ in the wavelength range from 2.0 to $6.5 \AA$. It represents currently the only available method in the sense of both good energy resolution and good spatial resolution.

\subsection{Transmission based crystal monochromators}

Transmission of the initial polychromatic neutron beam through a non-perfect monocrystal is conveniently given by the Gaussian function of the angle $\theta$ that corresponds to the neutron wavelength $\lambda$ according to the Bragg law. Using at least two crystals and changing the rotation angle of each crystal in respect to the initial beam direction enables an extraction of the wanted wavelength $\lambda$ with an arbitrary transmission value and a width bounded by the degree of crystals mosaicities. A sequence of several crystal pairs of different mosaicities can be employed to extract the wanted wavelength. The low mosaicity crystal pair is used to narrow the width of the extracted wavelength and higher mosaicity crystal pairs to remove the rest of the wavelength range. This method, first proposed by Tamaki [28] provides wavelength bands of low constant widths $(\Delta \lambda / \lambda \sim 1 \%-3 \%)$ in a broad wavelength range with the advantage of maintaining the initial beam direction.

\section{Experiment}

\subsection{Experimental set up}

Energy selective neutron radiography experiments were performed at two cold neutron imaging beam lines ICON, PSI 

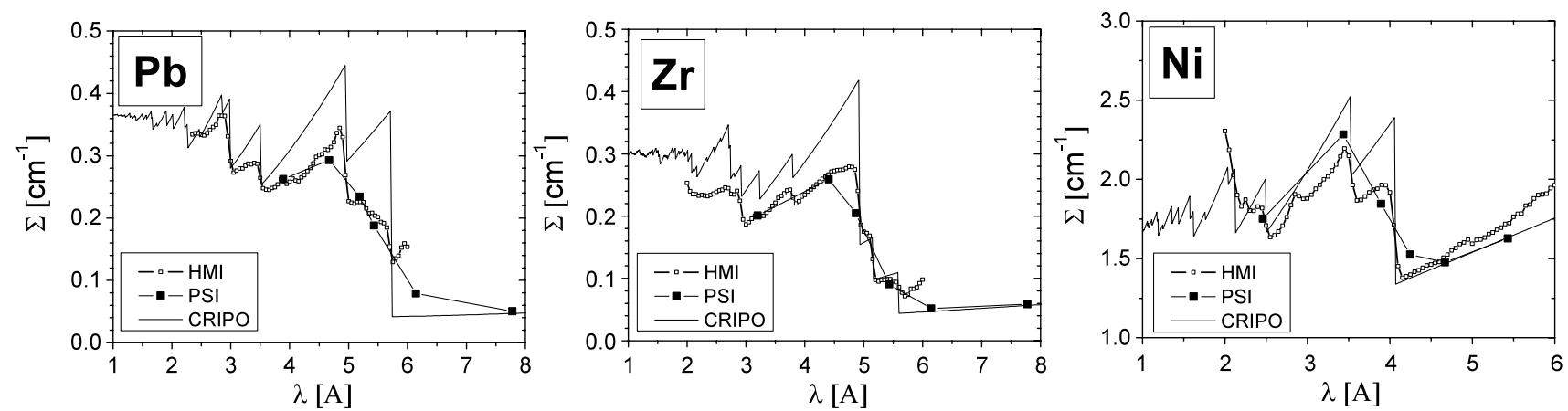

Fig. 4 Attenuation coefficients $(\Sigma)$ for $\mathrm{Pb}, \mathrm{Zr}$ and $\mathrm{Ni}$ polycrystalline materials obtained experimentally at ICON, PSI (solid symbols) and CONRAD, HMI (open symbols) compared to the analytically evaluated data (CRIPO code [29], solid lines)

Fig. 5 Maps of attenuation coefficients $(\Sigma)$ at $3.4,3.8,4.0$ and $4.4 \AA$ (from left) of the welded steel sample
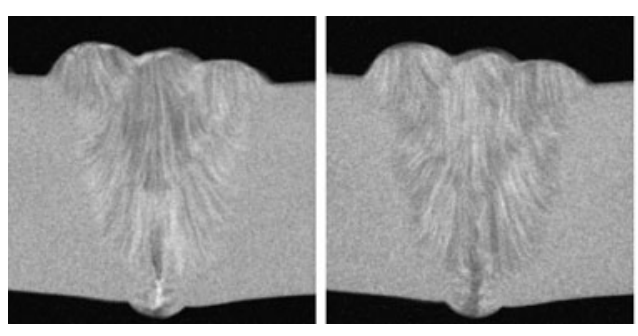

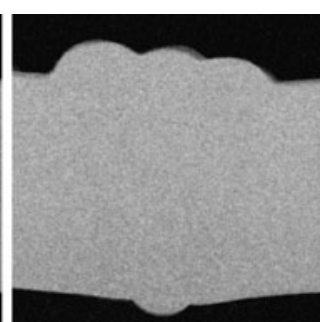

and CONRAD, HMI using the installed options for narrowing the broad polychromatic spectrums from the neutron sources. Both facilities provide currently the best conditions for energy selective radiography measurements. The velocity selector device employed at ICON enables wavelength bands widths of $\Delta \lambda / \lambda \sim 0.15$ and a double monochromator device at CONRAD wavelength bands of $0.1>$ $\Delta \lambda / \lambda>0.01$ widths. At both beam lines the same detection system developed at PSI was used, consisted of the cooled CCD camera with the $13.5 \mu \mathrm{m}$ pixel size and neutron absorbing $\mathrm{Gd}$ and $\mathrm{Li}$ scintillators that in combination with the good quality of the neutron beams (collimation, size, homogeneity and divergence) assured a high spatial resolution of $\sim 50 \mu \mathrm{m}$. The aim of the experiments was to derive total cross sections for neutron interaction with different polycrystalline materials ( $\mathrm{Al}, \mathrm{Zr}, \mathrm{Pb}, \mathrm{Cu}, \mathrm{Fe}$ and $\mathrm{Ni}$ ) and to visualize and quantify the highest possible energy and spatially resolved structural parameters of the complex material structure such welded steel entirely by neutron imaging.

\subsection{Experimental results and interpretation}

In the experiments with different materials, transmission through polycrystalline plates of different thicknesses was measured in the broad wavelength range between 2.0 and $6.0 \AA$ in $0.05 \AA$ steps at CONRAD and at six wavelengths at ICON. Collected images were corrected to the dark current offset of the CCD camera, background scattering and in some cases sample scattering. Sample scattering contribution to the radiographs taken at ICON for
Al was obtained by Monte Carlo simulations of the radiography experiment and the rest of the materials were not sample scattering corrected. Radiographs taken at CONRAD were for all materials corrected for sample scattering. Attenuation coefficients $\Sigma$ were evaluated from such corrected radiographs and compared to analytically evaluated values obtained with the CRIPO code [29]. Results for $\mathrm{Pb}, \mathrm{Zr}$ and $\mathrm{Ni}$ are shown in Fig. 4 as examples. Experimental values from both ICON and CONRAD beam lines agree well while the large differences exist in comparison to the CRIPO data in the certain wavelength ranges. This indicates the presence of textures in the measured samples that are especially strong, with the complete absence of the first Bragg edge, in $\mathrm{Al}, \mathrm{Cu}$ [30] and $\mathrm{Pb}$ (Fig. 4). Somewhat higher $\Sigma$ values of PSI measurements in comparison to $\Sigma$ HMI values are visible in $\mathrm{Cu}, \mathrm{Fe}$ [30] and $\mathrm{Ni}$ (Fig. 4) in some ranges. This indicates that the scattering contribution to the radiographs needs to be subtracted from the images, which was confirmed for the case of $\mathrm{Al}$ [30]. With this experiment, it is shown that neutron imaging provides structural parameters of crystalline materials.

The experiment with a steel weld was performed in the wavelength range between 2.9 and $4.4 \AA$ in $0.025 \AA$ wavelength steps at CONRAD, HMI with ICON, PSI CCD detection system. The imaged range of the sample includes the weld and the bulk material from both sides of the joint area. $\Sigma$ values were evaluated from the images obtained by division of the images taken with and without sample in the beam according to (12) for a weld thickness of $11.2 \mathrm{~mm}$ 
Fig. 6 Map of attenuation coefficients $(\Sigma)$ at $3.8 \AA$ (left $)$ and optical microscopy image (right) of the welded steel sample
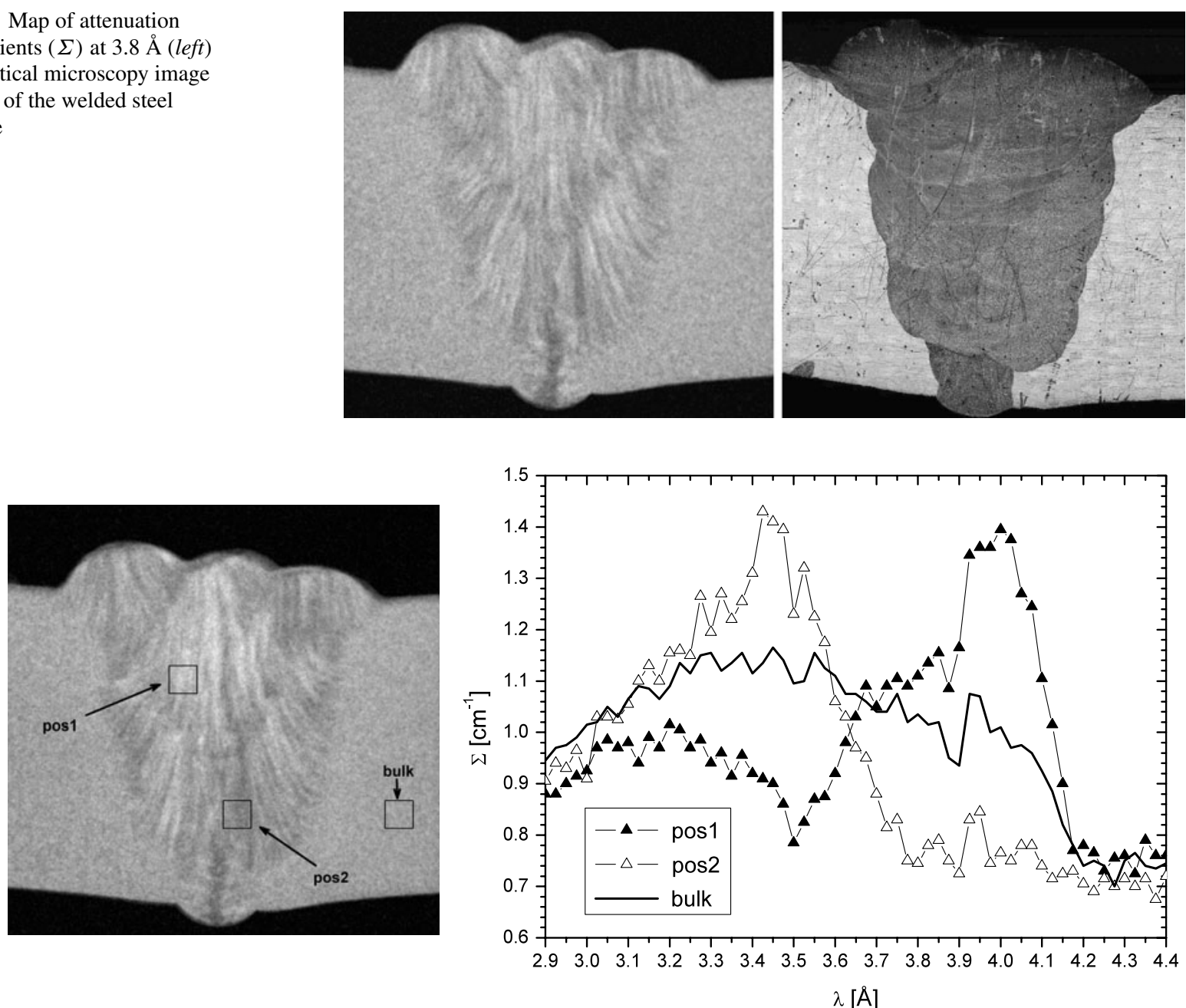

Fig. 7 Map of attenuation coefficients $(\Sigma)$ at an arbitrary wavelength with marked regions: pos1, pos2 and bulk (left) and the most probable $\Sigma$ values over the wavelength range from 2.9 to $4.4 \AA$ for the pos 1 , pos 2 and bulk regions (right) of the welded steel sample

in the beam direction. For each pixel, the most probable $\Sigma$ value in a Gaussian like distribution has been chosen as representative. The maps of $\Sigma$ values measured at 3.4, 3.8, 4.0 and $4.4 \AA$ are shown in Fig. 5. It can immediately be seen that long thin vertical stripes are visualized for each energy except $4.4 \AA$. Those imaged structures are perpendicular to the direction of the welding line, which can be seen from the comparison with the image taken by the standard optical microscopy of the polished and etched sample surface (Fig. 6). Further, the image contrast converts over the energy range with the highest contrast difference between images at 3.4 and $4.0 \AA$ (Fig. 5). Obtained maps of $\Sigma$ values over the weld area can be interpreted by the presence of different crystallographic textures in different weld regions. Each texture has a preferred direction in respect to the incident beam direction and thus attenuates the beam differently, which in turn changes the image contrast. For example, the energy dependence of $\Sigma$ for three different re- gions pos1, pos2 and bulk can be analyzed (Fig. 7). It can be seen that pos 1 and pos 2 regions show the opposite $\Sigma$ curves with peaks at 4.0 and $3.425 \AA$. This explains the opposite contrast on the images at 3.4 and $4.0 \AA$. Because at $4.0 \AA$ only reflections from (111) planes of steel are possible (the first Bragg edge for steel is at $4.169 \AA$ and the second at $3.6105 \AA$ [9]), according to (6), the peak at $4.0 \AA$ corresponds to the preferred crystallites orientation in the region pos 1 at angle $\sim 16^{\circ}$ in respect to the incident beam direction. The region pos 2 contains both crystallites with orientation at angle $\sim 35^{\circ}$ and those with orientation at angle $\sim 18^{\circ}$ because the peak in $\Sigma$ energy dependence at $3.425 \AA$ lies below the second Bragg edge (3.6105 $\AA$ [9]) and reflections from (200) planes become also possible. These results show that energy selective neutron imaging provides insight into the complex structures of materials, which is not available in a conventional polychromatic neutron beam approach. 


\section{Conclusion}

In the presented work it has been shown that with currently the best possible energy and spatial resolutions achieved simultaneously, energy selective neutron radiography presents an advanced technique for a determination of the crystalline structures in engineering materials. It has the capability to provide both: visualized and quantified maps of structural parameters and composition over the whole large sample area and thickness with the spatial resolution of $50 \mu \mathrm{m}$ and energy resolution of $10 \%>\Delta \lambda / \lambda>1 \%$ during the exposure time of less then only $60 \mathrm{~s}$. The modern digital detection system further enables a high quality data evaluation. Particular advancement is to be expected in employing neutron imaging for material research in the energy selective tomography mode where the full $3 \mathrm{D}$ information about the sample of a volume e.g. $3 \times 3 \times$ sample thickness $\mathrm{cm}^{3}$ can be obtained with the same energy and spatial resolutions within e.g. an hour of the exposure time. Such high performances of neutron imaging fit well with the demanding requirements from different industries, which in turn enable the further development in both scientific experimental and theoretical means.

Acknowledgements The authors would like to thank Dr. Nikolay Kardjilov and his team from Helmholtz-Zentrum, Berlin, Germany (former Hahn-Meitner Institut, Berlin, Germany) for collaboration and providing the beam time at CONRAD cold neutron imaging beam line.

\section{References}

1. P. Vontobel, E.H. Lehmann, R. Hassanein, G. Frei, Physica B 385386, 475 (2006)

2. G. Kühne, G. Frei, E. Lehmann, P. Vontobel, A. Bollhalder, U. Filges, M. Schild, Swiss Neutron News 28, 20 (2005)

3. N. Kardjilov, A. Hilger, I. Manke, M. Strobl, W. Treimer, J. Banhart, Nucl. Instrum. Meth. Phys. Res. A 542, 16 (2005)

4. B. Schillinger, J. Brunner, E. Calzada, Physica B 385-386, 921 (2006)
5. S. Baechler, N. Kardjilov, M. Dieric, J. Jolie, G. Kühne, E. Lehmann, T. Materna, Nucl. Instrum. Methods A 491, 481 (2002)

6. N. Kardjilov, S. Baechler, M. Bastürk, M. Dierick, J. Jolie, E. Lehmann, T. Materna, B. Schillinger, P. Vontobel, Nucl. Instrum. Methods A 501, 536 (2003)

7. N. Kardjilov, B. Schillinger, E. Steichele, Appl. Radiat. Isotopes 61, 455 (2004)

8. W. Treimer, M. Strobl, N. Kardjilov, A. Hilger, I. Manke, Appl. Phys. Lett. 89, 203504 (2006)

9. W. Kockelmann, G. Frei, E.H. Lehmann, P. Vontobel, J.R. Santisteban, Nucl. Instrum. Methods Phys. Res. A 578, 421 (2007)

10. J.R. Santisteban, L. Edwards, A. Steuwer, P.J. Withers, J. Appl. Crystallogr. 34, 289 (2001)

11. J.R. Santisteban, L. Edwards, M.E. Fitzpatrick, A. Steuwer, P.J. Withers, M.R. Daymond, M.W. Johnson, N. Rhodes, E.M. Schooneveld, Nucl. Instrum. Methods A 481, 255 (2002)

12. J.R. Santisteban, L. Edwards, V. Stelmukh, Physica B 385-386, 636 (2006)

13. A. Steuwer, P.J. Withers, J.R. Santisteban, L. Edwards, J. Appl. Phys. 97, 074903 (2005)

14. J.R. Santisteban, J. Appl. Crystallogr. 38, 934 (2005)

15. A.S. Tremsin, J.V. Vallerga, J.B. McPhate, O.H.W. Siegmund, W.B. Feller, L. Crow, R.G. Cooper, Nucl. Instrum. Methods Phys. Res. A 592, 374-384 (2008)

16. S. Vogel, A Rietveld-approach for the analysis of neutron time-offlight transmission data. Dissertation, University Kiel, 2000

17. G. Muhrer, T. Hill, F. Tovesson, E. Pitcher, Nucl. Instrum. Methods A 572, 866-873 (2007)

18. S.W. Lovesey, Theory of Neutron Scattering from Condensed Matter. Nuclear Scattering, vol. 1 (Clarendon Press, Oxford, 1986)

19. J.R. Granada, Z. Naturforsch. A 39a, 1160 (1984)

20. E.H. Lehmann, P. Vontobel, G. Frei, C. Brönnimann, Nucl. Instrum. Methods A 531, 228 (2004)

21. E. Lehmann, P. Vontobel, Appl. Radiat. Isotopes 61, 567 (2004)

22. N. Kardjilov, F. de Beer, R. Hassanein, E. Lehmann, P. Vontobel, Nucl. Instrum. Methods Phys. Res. A 542, 336 (2005)

23. R. Hassanein, E. Lehmann, P. Vontobel, Nucl. Instrum. Methods Phys. Res. A 542, 353 (2005)

24. R. Hassanein, Dissertation, ETHZ, 2006

25. D.B. Pelowitz (ed.), MCNPX User's Manual, Version 2.5.0, LACP-05-0369, Los Alamos National Laboratory, April 2005

26. H. Friedrich, V. Wagner, P. Wille, Physica B 156-157, 547 (1989)

27. E.H. Lehmann, P. Vontobel, G. Frei, J. Neutron Res. 13, 27 (2005)

28. M. Tamaki, Nucl. Instrum. Methods A 542, 32-37 (2005)

29. F. Kropff, J.R. Granada, Unpublished report (CAB-1977)

30. L. Josic, E.H. Lehmann, G. Frei, M. Tamaki, Nucl. Instrum. Methods A 605, 21-25 (2009) 